\title{
Coronagraphic near-IR photometry of AB Doradus C (Research Note)
}

\author{
A. Boccaletti ${ }^{1}$, G. Chauvin ${ }^{2}$, P. Baudoz ${ }^{1}$, and J.-L. Beuzit ${ }^{2}$ \\ 1 LESIA, Observatoire de Paris-Meudon 92195, Meudon, France \\ e-mail: [anthony.boccaletti; pierre.baudoz]@obspm. fr \\ 2 Laboratoire d'Astrophysique de l'Observatoire de Grenoble, Grenoble, France \\ e-mail: [gael.chauvin; jean-luc.beuzit]@obs.ujf-grenoble.fr
}

Received 20 December 2007 / Accepted 18 February 2008

\begin{abstract}
Context. Observations of low-mass companions for which the dynamical masses are well constrained help to improve the calibration of evolutionary models. Such observations thereby provide more confidence in the estimation of the mass of a companion using the photometric methods expected for the next generation of planet finder instruments.

Aims. The commissioning of a new coronagraph at the Very Large Telescope (VLT) was the occasion to test the performance of this technique on the well-known object AB Dor A and its $0.09 M_{\odot}$ companion AB Dor C. The purpose of this paper is to refine the photometric analysis on this object and to provide an accurate photometric error budget.

Methods. In addition to coronagraphy, we calibrated the residual stellar halo with a reference star. We used standard techniques for photometric extraction.

Results. The companion $\mathrm{AB}$ Dor $\mathrm{C}$ is easily detected at $0.185^{\prime \prime}$ from the primary star, and its magnitudes in $H$ and $K$ s are in agreement with an M 5.5 object, as already known from spectroscopic observations. However, these new measurements make the earlier $J$-band photometry less reliable. Finally, the comparison with evolutionary models supports an age of (75 \pm 25 ) Myr, contrary to previous analyses. These observations demonstrate that coronagraphic observations can be more efficient than direct imaging, not only to improve contrast, but also to provide a better photometric estimation as long as a good calibration of the stellar halo is achieved.
\end{abstract}

Key words. stars: individual: AB Dor - stars: low-mass, brown dwarfs - techniques: high angular resolution - methods: observational

\section{Introduction}

Since the last decade, the improvement of high angular resolution on large telescopes has made possible the discovery of faint companions with masses close to the planetary mass regime, 2M 1207 B (Chauvin et al. 2005a); DH Tau B (Itoh et al. 2005); GQLup B (Neuhäuser et al. 2005); AB Pic B (Chauvin et al. 2005b); and CHXR 73 B (Luhman et al. 2006). The precise determination of the mass of most of these companions is presently not possible as long as dynamical measurements are missing. However, an estimation of the mass can be obtained from photometric measurements via evolutionary models (Burrows et al. 1997; Chabrier et al. 2000). Calibration of these models on very low-mass objects, for which the mass is known from other techniques (radial velocity, astrometry), is highly desirable to prepare future instruments. The instruments SPHERE (Beuzit et al. 2006) and GPI (Macintosh et al. 2006) will precisely use broadband differential imaging or low-resolution spectroscopy to carry out statistical analysis on extrasolar planets. Hence, accurate mass estimation is critical in this context and a strong effort has been made to refine atmospheric models of giant planets and brown dwarfs.

Young, nearby associations are well suited to the identification and the follow-up observations of young dynamical mass calibrators, such as the tight binaries HD 98800 (Boden et al. 2005) and TWA5 Aab (Konopacky et al. 2007) of the TW Hydrae association. Very recently, much attention has been paid to the hierachical quadruple system AB Dor, a member of the eponymous comoving group identified by
Zuckerman et al. (2004). The brightest component AB Dor A was first known as a variable star featuring variation of $0.09 \mathrm{mag}$ in the $V$ band and flares of 0.05 mag near its maximum (Innis et al. 1985). It was then recognized as a rapidly-rotating spotted star and was intensively studied as such in the 1980s. Accurate parallax obtained with Hipparcos $(\pi=(6.92 \pm 0.54)$ mas, $d=$ $(14.9 \pm 0.12)$ pc; Perryman et al. 1997) allowed Wichmann et al. (1998) to derive a spectral type of K1, while it was previously thought to be a post-T Tauri star.

At 9.0" North, the physical companion AB Dor B (Lim 1993; Guirado et al. 2006) is resolved as a tight $\left(\Delta=0.070^{\prime \prime}\right)$ binary by Close et al. (2005). The object AB Dor $C$ is the fourth component of this young quadruple system, discovered thanks to the reflex motion induced on AB Dor A detected with Very Long Baseline Interferometry and Hipparcos observations (Guirado et al. 1997). Close et al. (2005) have refined the mass estimation of $\mathrm{AB}$ Dor $\mathrm{C}$ to $(0.090 \pm 0.005) M_{\odot}$ (confirmed later by Guirado et al. 2006). A first attempt to image this close and low-mass companion with ADONIS at the $3.6 \mathrm{~m}$ telescope of La Silla (ESO) was unsuccessful due to the lack of angular resolution (Boccaletti et al. 2001). Close et al. (2005) finally resolved $\mathrm{AB}$ Dor $\mathrm{C}$ at $0.156^{\prime \prime}$ from A using VLT/NACO. They measured the near IR absolute magnitudes (see Table 2) and a spectral type M $8 \pm 1$ for this faint companion. The $J$ and $H$-brightnesses and the deduced effective temperature were found to be inconsistent with evolutionary models, considering an age estimate of $50_{-20}^{+30} \mathrm{Myr}$ based on different youth indicators. In conclusion, Close et al. (2005) suggested that theoretical 
models are actually underestimating the mass in the young age and low-mass regime.

Luhman \& Potter (2006) revised the JHK photometry uncertainties and the spectral type estimation of Close et al. (2005) based on the same data set. Using, in addition, a different age estimate of 75-150 Myr for the AB Dor association (Luhman et al. 2005), they concluded that there was currently no disagreement between models and data. Recent VLT/SINFONI spectra in $H K$-bands enable Close et al. (2007) to derive a spectral type M $5.5 \pm 1$, which confirms the conclusions of Luhman \& Potter (2006). This prolific system illustrates the difficulty of testing evolutionary model predictions without accurate observables (effective temperature and luminosity) and robust age estimate.

In 2006, a proposal to combine the Simultaneous Differential Imaging (SDI) mode of NACO (the Nasmyth Adaptive Optics System and Near-Infrared Imager and Spectrograph) with a 4 Quadrant Phase Mask coronagraph (Rouan et al. 2000) was approved by European Southern Observatory (ESO). During the commissioning run we collected data on AB Dor A and C (Sect. 2) and here, we present the results of our $H$ and $K$ s photometric analysis (Sect. 3) together with a detailed estimation of error bars (Sect. 4). Results are discussed in Sect. 5.

\section{Observations}

We carried out observations as part of a commissioning run on February 16th, 2007 at ESO/Paranal. The AO-assisted near-IR camera NAOS-CONICA named NACO (Rousset et al. 2003; Lenzen et al. 2003) was equipped with a new set of two 4 Quadrant Phase Masks (Rouan et al. 2000) to replace the old one (Boccaletti et al. 2004). These two masks are operating respectively in the $K$ s and $H$ bands, the latter being compatible with the Simultaneous Differential Imager (SDI) provided by the University of Arizona and the Max Planck Institute of Heidelberg (Lenzen et al. 2004).

We observed AB Dor $(V=6.93, H=4.845, K=4.686$, $\mathrm{Sp}=\mathrm{K} 1 \mathrm{III})$ with the 4 Quadrant Phase Mask (4QPM) in two filters. We obtained $600 \mathrm{~s}($ DIT $=1 \mathrm{~s}$, NDIT $=100$, Ncycle $=6)$ of data in the $K \mathrm{~s}$ band $(\lambda=2.18 \mu \mathrm{m}, \Delta \lambda=0.35 \mu \mathrm{m})$ on the target and a similar integration time on a reference star (HD 41371) chosen with the same visible and IR fluxes $(V=7.10, K=$ 4.724, $\mathrm{Sp}=\mathrm{K} 0 \mathrm{III}$ ) and observed at the same parallactic angle. This optimal observing strategy preserves the orientation of the telescope pupil with respect to NACO, and therefore, reduces the differential aberrations between the star and its reference as the matching of spider spikes in the two images. In addition, we observed AB Dor with SDI (the 4QPM being installed in the beam) for $936 \mathrm{~s}($ DIT $=8 \mathrm{~s}$, NDIT $=13$, Ncycle $=9)$. Instead of a reference star, the calibration of the speckled halo is obtained simultaneously in different filters $(\lambda=1.575,1.600$, $1.625 \mu \mathrm{m}, \Delta \lambda=0.025 \mu \mathrm{m}$ ). However, a second level of calibration is required to reduce the impact of differential aberrations and the speckle chromaticity (as the phase varies with wavelength, Marois et al. 2000). For this purpose, we obtained two observations with the field of view rotated by $60^{\circ}$. Differential aberrations are assumed static in this case.

Coronagraphic observations with NACO are preceded with an acquisition template that provides an out-of-mask PSF to be used as a photometric reference. However, this reference is obtained with a different setup than coronagraphic frames. Because AB Dor is a bright object, a Neutral Density (ND) is needed to avoid detector saturation (for $K \mathrm{~s}$ band data only), and as default a full aperture stop (Full) is used instead of a stopped aperture
(Full_uszd) as for coronagraphic templates. Photometric measurements have to be corrected from these values.

Seeing conditions and AO correction were good. The average seeing was $(0.91 \pm 0.13)^{\prime \prime}$ for the $K$ s observing block and $(0.78 \pm 0.12)^{\prime \prime}$ for the SDI data while the coherent energy measured from residual slopes of the AO system was, respectively, $(46 \pm 5) \%$ and $(52 \pm 4) \%$.

\section{Data reduction and photometric measurements}

We processed the data with standard reduction routines to correct for bad pixels, flat field uniformity and to subtract an average sky background. We obtained flat fields without the coronagraph in the beam, although it would have been better to reduce the presence of dust particles on the substrate. However, the field of view slightly drifted in front of the detector plane (as the instrument rotates at the Nasmyth focus), making the precise registration of dust features on the coronagraph substrate impossible.

We corrected for the ND attenuation on the $K$ s data (a factor of 89) and for the difference of pupil stop (a factor of 0.808 estimated from geometrical assumptions) to build a master normalized PSF. The individual coronagraphic cycles were then coadded for the star and the reference separately so as to provide two images. Recentering was not required. At this stage, the companion is yet visible (Figs. 1 and 2, left), but we needed the subtraction of the reference star to remove the PSF halo and to accurately measure the photometry. To estimate the intensity factor between the coronagraphic image of the star and that of the reference, we considered several methods: total intensity ratio, balance of positive and negative fluxes, balance of positive and negative pixels, and minimization of the total residual intensity. We adopt the last method as a baseline paying attention to the presence of the companion to avoid a bias. We found an intensity factor of $1.04 \pm 0.01$ with small dispersion between methods and produced a subtracted coronagraphic image (Fig. 1, right).

Once the stellar contribution is removed at the companion location a thorough estimation of the companion intensity becomes possible. Here also, we compared several methods like aperture photometry, PSF fitting with a 2D Gaussian, PSF to companion maximum intensity ratio, and minimization of the residual after PSF subtraction (on the companion). In the two first cases, the companion intensity is integrated in a limited aperture (a few pixels in radius) and a correction of $-0.1 \mathrm{mag}$ is needed to account for the intensity in the PSF wings (comparison of encircled energy). As a baseline, we used the minimization to derive the average photometry.

In the particular case of SDI data, two wavelengths subtraction is inappropriate since $\mathrm{AB}$ Dor $\mathrm{C}$ does not contain methane and the bifurcation point (the distance at which the companion image in the rescaled frame falls at different pixels than in the unrescaled frame, Thatte et al. 2007) is located at 3.2". However, observational procedure with SDI requires the acquisition of a field-rotated image of the same star to correct for the differential static aberrations and the chromatic aberrations inherent to the SDI technique. Therefore, we measured $H$ band flux of $\mathrm{AB}$ Dor $\mathrm{C}$ with the field-rotated image as a reference star both in a single SDI filter $(\Delta \lambda=0.025 \mu \mathrm{m})$ and with the combination of all filters $(\Delta \lambda=0.075 \mu \mathrm{m})$ to approach the broadband magnitude. Hence, two values are provided for the $H$ band magnitudes that we note: $m_{H_{1 \lambda}}$ and $m_{H_{3 \lambda}}$.

As a result, we found the following magnitude differences: $\Delta m_{H_{1 \lambda}}=4.71, \Delta m_{H_{3 \lambda}}=4.62$ and $\Delta m_{K \mathrm{~s}}=4.56$. The angular separation is $0.185^{\prime \prime}$ consistent with the orbital solution presented in Nielsen et al. (2005). However, it was not the goal of this paper 

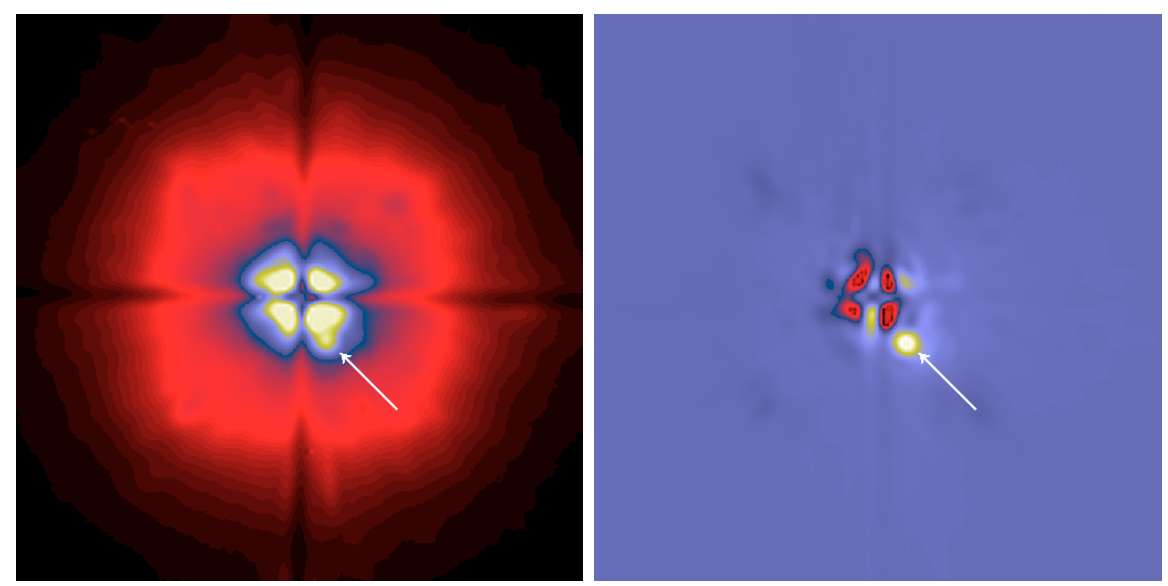

Fig. 1. Coronagraphic image of $\mathrm{AB}$ Dor obtained in the $K$ s filter (Left) and the same image subtracted with a reference star (Right). The field of view is $2^{\prime \prime}$. North is up, East is left. Arbitrary false colors are intended to enhance the companion visibility.
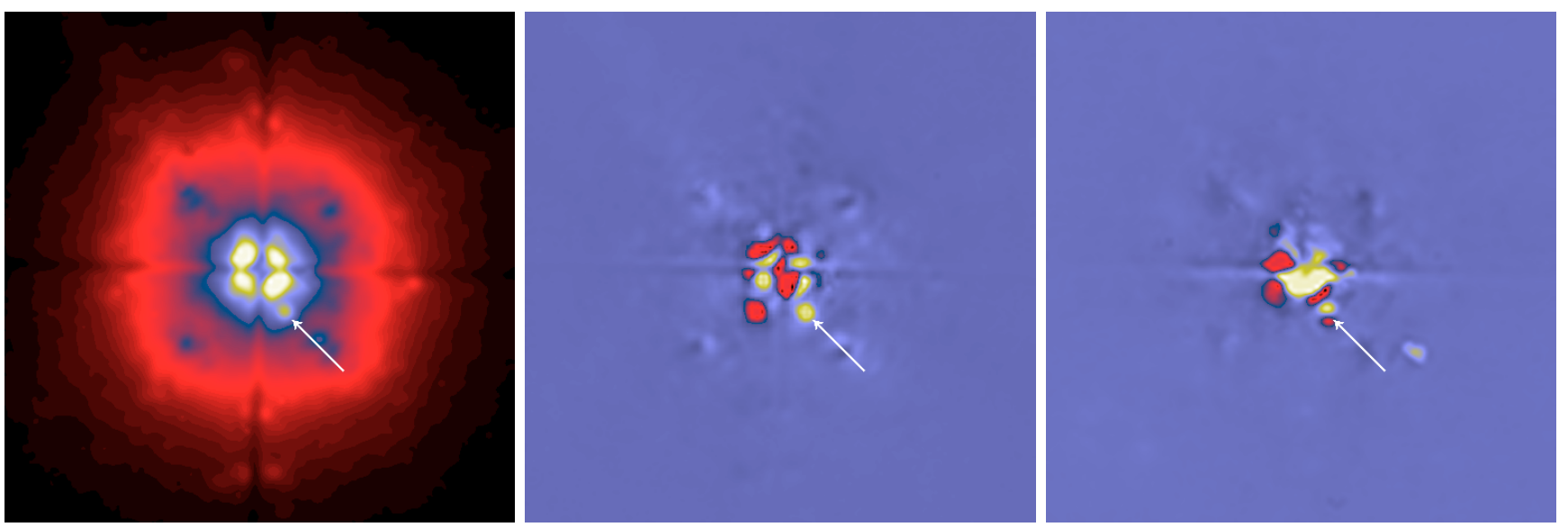

Fig. 2. Coronagraphic image of AB Dor obtained with SDI (Left) and the same image subtracted with a field-rotated AB Dor image (Middle) compared to the 2 wavelengths subtraction (Right). Subtracted frames show a positive image of the companion (yellow) and a negative one (red). The SDI frame is not exploitable because of the small angular separation making the positive and negative component self-subtracting. The field of view is $2^{\prime \prime}$. North is up, East is left. Arbitrary false colors are intended to enhance the companion visibility.

to discuss the astrometry of the companion since it was already characterized from Hipparcos data and confirmed by the aforesaid papers. In addition, the presence of the coronagraph makes the estimation of the astrometry less accurate unless appropriate techniques are considered (Marois et al. 2006).

\section{Photometric errors}

We identified several sources of errors in the photometric extraction that we analyze in this section to derive error bars:

- the pupil Lyot stop correction made on the PSF flux. As mentioned, a geometrical comparison of the Full aperture and the Full_uszd aperture (undersized by 10\%) leads to a correction of 0.808 . However, a photometric measurement obtained in a previous observing run suggests a value of 0.775 . Then, an uncertainty of $4 \%$ was considered;

- the ND correction made on the PSF flux (only for data in $K \mathrm{~s})$. Our measurement in the $K \mathrm{~s}$ band yields an attenuation factor of $89 \pm 3.6$

- intensity factor between the coronagraphic image of the star and that of the reference. The variety of method we used to measure this parameter is providing a good estimate of the uncertainty. The precision achieved is $1 \%$;
Table 1. Photometric uncertainties in magnitude.

\begin{tabular}{lcc}
\hline \hline Sources of error & $H$ & $K \mathrm{~s}$ \\
\hline pupil stop & 0.05 & 0.05 \\
ND & - & 0.06 \\
intensity factor & 0.05 & 0.04 \\
method for extraction & 0.13 & 0.13 \\
aperture size & 0.04 & 0.01 \\
distance & 0.03 & 0.03 \\
2MASS & 0.03 & 0.02 \\
filters conversion & 0.03 & 0.01 \\
\hline total quadratic error & 0.16 & 0.16 \\
\hline
\end{tabular}

- photometric extraction. Here again, we used a variety of methods to consolidate the result. However, we identified it as the major source of uncertainty in our measurement. The dispersion between methods is of about $0.13 \mathrm{mag}$;

- aperture size, when aperture photometry is used for photometric extraction. The radius of the aperture photometric mask is set to $1.22 \lambda / D_{\text {lyot }}, D_{\text {lyot }}$ being the diameter of the Lyot stop instead of that of the telescope $(7.2 \mathrm{~m}$ instead of $8 \mathrm{~m}$ ). A variation of 1 pixel on this radius provides the error bar. Although aperture photometry is not used to extract the photometry (see Sect. 3) it actually enters in the error term 
Table 2. Photometry of AB Dor, A being the primary and C the companion converted into the 2MASS system.

\begin{tabular}{|c|c|c|c|c|c|c|c|}
\hline$\overline{\text { UT date }}$ & $\begin{array}{l}M_{J} \\
(\mathrm{mag})\end{array}$ & $\begin{array}{l}M_{H} \\
(\mathrm{mag})\end{array}$ & $\begin{array}{l}M_{K \mathrm{~s}} \\
(\mathrm{mag})\end{array}$ & $\begin{array}{l}\Delta \\
\text { (mas) }\end{array}$ & 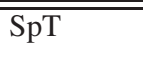 & $\begin{array}{l}T_{\text {eff }} \\
(\mathrm{K})\end{array}$ & "References \\
\hline $\begin{array}{l}07.01 .2005 \\
24.01 .2006 \\
16.02 .2007 \\
16.02 .2007\end{array}$ & $\begin{array}{l}9.89_{-0.24}^{+0.19} \\
9.85_{-0.46}^{+0.32}\end{array}$ & $\begin{array}{l}9.17_{-0.15}^{+0.13} \\
9.31_{-0.31}^{+0.24}\end{array}$ & $\begin{array}{l}8.58_{-0.15}^{+0.12} \\
8.92_{-0.37}^{+0.28} \\
8.63 \pm 0.17 \\
8.38 \pm 0.16\end{array}$ & $\begin{array}{l}156 \\
156 \\
220 \\
200 \\
185\end{array}$ & $\begin{array}{l}\text { M } 8 \pm 1 \\
\text { M } 6 \pm 1 \\
\text { M } 5.5 \pm 1\end{array}$ & $\begin{array}{r}2600_{-150}^{+150} \\
2840_{-120}^{+170} \\
2925_{-140}^{+170}\end{array}$ & $\begin{array}{l}\text { Close et al. (2005) } \\
\text { Luhman \& Potter (2006) } \\
\text { Close et al. (2007) } \\
\text { Thatte et al. (2007), Close et al. (2007) } \\
\text { this work } \\
\text { this work }\end{array}$ \\
\hline
\end{tabular}

"method of extraction", but has a wavelength dependence according to the PSF sampling;

- the star distance which is known to an accuracy of $0.12 \mathrm{pc}$;

- the 2MASS photometric uncertainty for the primary star magnitude;

- the conversion between systems of magnitude (NACO to 2MASS to CIT).

Table 1 gives the correspondence of these uncertainties in magnitude. To derive the $\mathrm{AB}$ Dor $\mathrm{C}$ absolute magnitudes in the $H$ and $K$ s 2MASS system, we have estimated the NACO to 2MASS filters transformations based on the primary and secondary spectral types and the filter transmission curves. The transformations found are $(-0.02 \pm 0.03) \mathrm{mag},(-0.04 \pm 0.03) \mathrm{mag}$, and $(0.00 \pm$ $0.01)$ mag in $H_{1 \lambda}, H_{3 \lambda}$, and $K$ s respectively. Assuming errors are added quadratically and a distance of $d=(14.9 \pm 0.12) \mathrm{pc}$, the new $H$ - and $K$ s-absolute magnitudes of AB Dor C were obtained with uncertainties and are given with the results of previous works in Table 2.

\section{Discussion}

Our new $H$ and $K$ s measurements of $\mathrm{AB}$ Dor $\mathrm{C}$ and the derived $H-K \mathrm{~s}=0.29 \pm 0.23$ color are consistent with the recent M $5.5 \pm 1$ spectral type estimation of Close et al. (2007) (using spectroscopic analysis rather than photometry). These new measurements also weaken the reliability of the $J$-band photometry of Close et al. (2005) and Luhman \& Potter (2006) obtained in poor atmospheric conditions and that "may be systematically too faint" (Close et al. 2007). The $J-K$ s would be indeed even redder than before and surprisingly red compared to M $5.5 \mathrm{ob}-$ jects of the Pleiades. Although not impossible, the existence of an IR excess for $\mathrm{AB}$ Dor $\mathrm{C}$ seems to be unlikely as $\mathrm{AB}$ Dor $\mathrm{A}$ (K1), Ba (M $3.5 \pm 1.5)$, and $\mathrm{Bb}(\mathrm{M} 4.5 \pm 1.5)$ do not present abnormal $(J-K s)$ colors.

Our new $H$ and $K$ s absolute magnitudes of AB Dor C (color corrected again between the 2MASS and CIT systems) are compared with previous measurements and over-plotted on evolutionary model predictions of Chabrier et al. (2000) for a dynamical mass of $(0.09 \pm 0.005) M_{\odot}$ (Guirado et al. 2006). For the following analysis, we have considered two scenarii of ages for the AB Dor system, $(75 \pm 25) \mathrm{Myr}$ and $(120 \pm 20) \mathrm{Myr}$, as the determination of this fundamental parameter is still debated.

The first age estimate of $~ 50$ Myr was given by Zuckerman et al. (2004) for the whole AB Dor association. We used multiple youth indicators, such as $\mathrm{H}_{\alpha}$ emission, strong lithium $6708 \AA$ absorption, large $v \sin i$, large X-ray flux, and a location above the main sequence of a color $(V-K)$ magnitude $\left(M_{K}\right)$ diagram for three mid-M type members. Close et al. (2005) and Nielsen et al. (2005) estimated a more precise age range to $50_{-20}^{+50} \mathrm{Myr}$ based on comparisons with other young clusters properties. Similar analysis led López-Santiago et al. (2006) to confirm this age

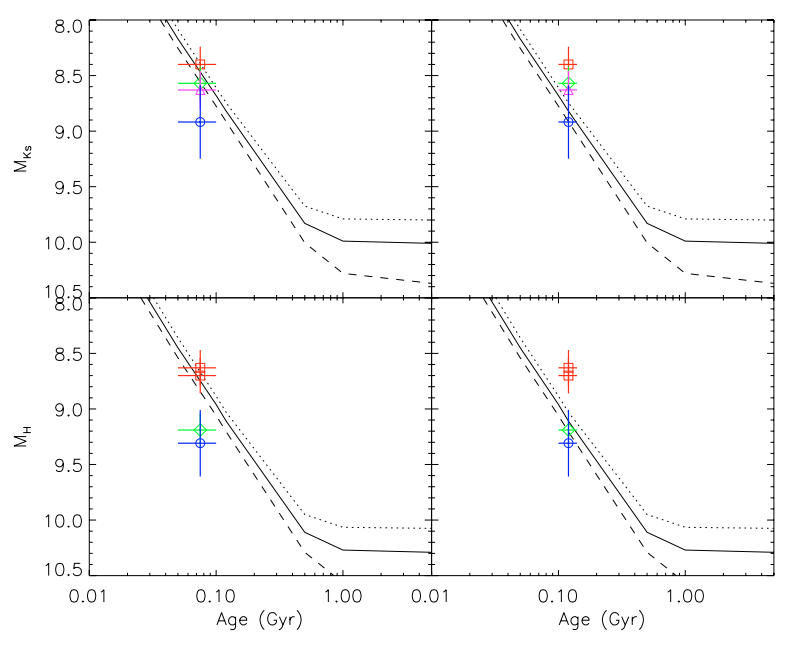

Fig. 3. Interpolated evolutionary tracks of Chabrier et al. (2000) for 0.085 (dashed), 0.090 (solid) and 0.095 (dotted) solar masses in the range 0.01-10 Gyr compared with our photometric measurements (red box) those of Close et al. (2005) (green diamond) and Close et al. (2007) (purple triangle) and those of Luhman \& Potter (2006) (blue circle). Upper and lower plots are respectively for $K \mathrm{~s}$ and $H$ photometry (in the CIT system of magnitudes). The left plots are for the first age scenario (75 $\pm 25 \mathrm{Myr})$ and the right plots for the second (120 $\pm 20 \mathrm{Myr})$.

estimate. More recently, Janson et al. (2007) focused their agedating criteria on the the two M dwarfs AB Dor Ba and Bb, comparing their effective temperatures to evolutionary track predictions, which are relatively robust for low-mass stars. However, uncorrect spectral types for both components led them to over constrain the upper age limit for this system. We show model predictions in Fig. 3 (left), and compared to our measurements for an age of $(75 \pm 25)$ Myr. Contrary to the measurements of Close et al. (2005) and Luhman \& Potter (2006) obtained in less favorable conditions (smaller angular separation and lower AO correction), our results are clearly in good agreement with evolutionary tracks in both $H$ and $K$ s and would confirm the validity of evolutionary models in this range of masses and ages.

Using comparable age-dating indicators, Luhman et al. (2005) derived a second estimate of 75-150 Myr for the AB Dor association. Moreover, like Innis et al. (1986), they have suggested that the AB Dor association might belong to the Pleiades supercluster sharing common kinematic origin. Based on the 3D dynamical evolution analysis of the AB Dor association and the Pleiades, Ortega et al. (2007) obtained a similar age of $(120 \pm 20)$ Myr consistent with both groups being coeval. If we now consider this age-scenario shown in Fig. 3 (Right), our measurements are not well fitted in both bands by the model predictions. The model predictions actually underestimate the observed luminosity of AB Dor C by a factor of $\sim 1.6$ (0.5 mag), 
and thus overestimate its mass (opposite of what was found by Close et al. 2005).

Future age characterization and confirmation of our photometric results should help to draw a robust conclusion to estimate the accuracy of the model predictions in this range of ages and masses. More interestingly, the results presented here evidence the ability of broadband photometry combined with coronagraphy to retrieve the actual companion mass, providing optical quality is met to allow the use of a coronagraph. This conclusion is important in the context of planet finder instruments like SPHERE and GPI, using differential imaging observations of some planetary spectral features and for which planetary masses will be assessed via the comparison of spectral contrasts and evolutionary models.

Acknowledgements. We would like to thanks the ESO team at the telescope who support the commissioning of the coronagraph and also R. Lenzen for the installation of the masks inside CONICA and for his suggestions as a referee.

\section{References}

Beuzit, J.-L., Feldt, M., Dohlen, K., et al. 2006, The Messenger, 125, 29 Boccaletti, A., Moutou, C., Mouillet, D., Lagrange, A.-M., \& Augereau, J.-C. 2001, A\&A, 367, 371

Boccaletti, A., Riaud, P., Baudoz, P., et al. 2004, PASP, 116, 1061

Boden, A. F., Sargent, A. I., Akeson, R. L., et al. 2005, ApJ, 635, 442

Burrows, A., Marley, M., Hubbard, W. B., et al. 1997, ApJ, 491, 856

Chabrier, G., Baraffe, I., Allard, F., \& Hauschildt, P. 2000, ApJ, 542, 464

Chauvin, G., Lagrange, A.-M., Dumas, C., et al. 2005a, A\&A, 438, L25

Chauvin, G., Lagrange, A.-M., Zuckerman, B., et al. 2005b, A\&A, 438, L29

Close, L. M., Lenzen, R., Guirado, J. C., et al. 2005, Nature, 433, 286

Close, L. M., Thatte, N., Nielsen, E. L., et al. 2007, ArXiv Astrophysics e-prints
Guirado, J. C., Martí-Vidal, I., Marcaide, J. M., et al. 2006, A\&A, 446, 733 Guirado, J. C., Reynolds, J. E., Lestrade, J.-F., et al. 1997, ApJ, 490, 835 Innis, J. L., Coates, D. W., Thompson, K., \& Robinson, R. D. 1985, Proc. Astron. Soc. Austr., 6, 156

Innis, J. L., Thompson, K., \& Coates, D. W. 1986, MNRAS, 223, 183

Itoh, Y., Hayashi, M., Tamura, M., et al. 2005, ApJ, 620, 984

Janson, M., Brandner, W., Lenzen, R., et al. 2007, A\&A, 462, 615

Konopacky, Q. M., Ghez, A. M., Duchêne, G., McCabe, C., \& Macintosh, B. A. 2007, AJ, 133, 2008

Lenzen, R., Hartung, M., Brandner, W., et al. 2003, in SPIE, 4841, 944

Lenzen, R., Close, L., Brandner, W., Biller, B., \& Hartung, M. 2004, in SPIE, 5492, 970

Lim, J. 1993, ApJ, 405, L33

López-Santiago, J., Montes, D., Crespo-Chacón, I., \& Fernández-Figueroa, M. J. 2006, ApJ, 643, 1160

Luhman, K. L., \& Potter, D. 2006, ApJ, 638, 887

Luhman, K. L., Stauffer, J. R., \& Mamajek, E. E. 2005, ApJ, 628, L69

Luhman, K. L., Wilson, J. C., Brandner, W., et al. 2006, ApJ, 649, 894

Macintosh, B., Graham, J., Palmer, D., et al. 2006, in Advances in Adaptive Optics II, ed. B. L. Ellerbroek, \& D. Bonaccini Calia, Proc. SPIE, 6272, $62720 \mathrm{~L}$

Marois, C., Doyon, R., Racine, R., \& Nadeau, D. 2000, PASP, 112, 91

Marois, C., Lafrenière, D., Macintosh, B., \& Doyon, R. 2006, ApJ, 647, 612

Neuhäuser, R., Guenther, E. W., Wuchterl, G., et al. 2005, A\&A, 435, L13

Nielsen, E. L., Close, L. M., Guirado, J. C., et al. 2005, Astron. Nachr., 326, 1033

Ortega, V. G., Jilinski, E., de La Reza, R., \& Bazzanella, B. 2007, MNRAS, 377 , 441

Perryman, M. A. C., Lindegren, L., Kovalevsky, J., et al. 1997, A\&A, 323, L49 Rouan, D., Riaud, P., Boccaletti, A., Clenet, Y., \& Labeyrie, A. 2000, PASP, 112 , 1479

Rousset, G., Lacombe, F., Puget, P., et al. 2003, in SPIE, 4839, 140

Thatte, N., Abuter, R., Tecza, M., et al. 2007, MNRAS, 378, 1229

Wichmann, R., Bastian, U., Krautter, J., Jankovics, I., \& Rucinski, S. M. 1998, MNRAS, 301, L39

Zuckerman, B., Song, I., \& Bessell, M. S. 2004, ApJ, 613, L65 